\title{
FOURIER SINE TRANSFORM METHOD FOR SOLVING THE CERRUTTI PROBLEM OF THE ELASTIC HALF PLANE IN PLANE STRAIN
}

\author{
CHARLES CHINWUBA IKE - Lecturer, PhD. Department of Civil Engineering, Enugu State University of \\ Science and Technology, Enugu, Enugu State, Nigeria, e-mail: charles.ike@esut.edu.ng
}

\begin{abstract}
The Fourier sine transform method was implemented in this study to obtain general solutions for stress and displacement fields in homogeneous, isotropic, linear elastic soil of semiinfinite extent subject to a point load applied tangentially at a point considered the origin of the half plane. The study adopted a stress based formulation of the elasticity problem. Fourier transformation of the biharmonic stress compatibility equation was done to obtain bounded stress functions for the elastic half plane problem. Stresses and boundary conditions expressed in terms of the Boussinesq-Papkovich potential functions were transformed using Fourier sine transforms. Boundary conditions were used to obtain the unknown constants of the stress functions for the Cerrutti problem considered; and the complete determination of the stress fields in the Fourier transform space. Inversion of the Fourier sine transforms for the stresses yielded the general expressions for the stresses in the physical domain space variables. The strain fields were obtained from the kinematic relations. The displacement fields were obtained by integration of the straindisplacement relations. The solutions obtained were identical with solutions in literature obtained using Cerrutti stress functions.
\end{abstract}

Keywords: Fourier sine transform method, elastic half plane, Boussinesq-Papkovich potential functions, biharmonic stress compatibility equation, stress fields, displacement fields

\section{Introduction}

The problem of finding stress and displacement variations within an elastic half plane due to point or distributed loads applied to the surface or inside the half plane belongs to the classical mathematical theory of elasticity and can be considered a two dimensional (2D) specialization of the elastic half space problem [1, 2, 3, 4, 5]. Such elastic half plane and half-space problems have extensive applications in the analysis and design of foundation structures, pile foundations, and geotechnical engineering $[6,7]$. The elastic half plane/space material may be considered isotropic or anisotropic, homogeneous or heterogeneous, linear elastic or non-linear elastic. In general, the characterisation of the half plane material determines the complexity and rigour of the elastic half plane problem. Anisotropic, heterogeneous, non linear elastic half plane problems are usually very complicated, and rigorous in both formulation and their solution. The type of load also determines the complexity and rigour of the elastic half plane/or half-space problem; and gives rise to several types of the half space problem. When the load is a point load applied inside the elastic half space, the problem becomes a Mindlin problem. For point load applied vertically at the origin $(0,0,0)$ of the elastic half space, the problem is called the Boussinesq problem. The Cerrutti problem is concerned with a point load applied tangentially in the $x-$ direction at the origin of an elastic half plane or half space. The Boussinesq, Mindlin and Cerrutti problems are fundamental problems in that their solutions can be used as Green functions to generate solutions for distributed loads on the half plane or half space.

The elastic half plane and half-space problems are formulated by the use of the fundamental equations of elasticity theory; namely: the differential equations of equilibrium, the material constitutive laws and the six strain displacement relations (also called the geometric relations of strain) subject to the compatibility equations and the traction and deformation boundary conditions. The number, type and complicated nature of the governing field equations of the 
elastic half space theory for a rigorous mathematical formulation and analytical solution are quite unwieldy and involve extensively intensive and rigorous advanced mathematical methodologies for analysis and solution [8].Hence, three basic methods have been used in order to provide a simplified formulation of elasticity problems. They are: the displacement method, the stress method and the mixed method [9, 10, 11].

In the displacement based methods, the three sets of fundamental requirements of the differential equations of equilibrium, the generalised Hooke's stress - strain laws, and the kinematic relations are reformulated in terms of the displacement components as the only unknown primary variables. This leads to a reduction in the number and complexity of the governing field equations from a set of 15 equations in terms of both stresses, strains and displacements to a set of three coupled equations in terms of the three displacement components. The displacement formulation was presented by Navier, Love, Lame, Boussinesq, Papkovich and Cerrutti.

In stress based elasticity problem formulations, the set of fifteen partial differential equations involving equilibrium, geometric relations and material constitutive laws are simultaneously reformulated such that unknown strains and displacements are eliminated from the expressions, and the Cauchy stresses become the only unknown primary variables [9-11]. This has the merit of reduction in the member and complication of the governing field equations for three dimensional problem from fifteen to six equations in terms of the three components of normal stresses and the three components of shear stresses. Stress based formulations of elasticity problems were presented by Michell, Love, Boussinesq, Papkovich, Beltrami, Airy, Morera, and Maxwell. The mixed formulation expresses the governing field equations in reformulated form in terms of some components of the Cauchy stress and some displacement components; as the primary unknown variables $[12,13]$.

This work employs the stress based formulation approach. The mathematical simplifications offered by the reformulations of the general elasticity problem have resulted in the derivations of stress and displacement functions that apriori satisfy the governing equations in stress based and displacement based formulations [10,11,14]. The stress and displacement functions provide more simplifications to the solutions of elasticity problems to the problem of finding suitable stress and displacement functions that satisfy the boundary conditions of traction, loading and displacement for the particular problem. This thus reduces the dimensionality of the general problem of the mathematical theory of elasticity; particularly for the half plane and half space problems.

In this work, the Boussinesq - Papkovich stress (displacement) potential functions are used in the method of Fourier sine transformation to determine the stress fields and displacement fields in a half plane due to a point load $Q_{0}$ acting at the origin and tangentially applied in the positive $\mathrm{x}$-direction. This problem was first solved by Valentino Cerrutti who considered a linearly elastic isotropic half space region subjected to the action of a concentrated load acting at the origin and applied tangentially to the boundary surface $z=0$. Padio-Guidugli and Favata [12] considered a variant of the Cerrutti problem where a distributed tangent load is applied with constant magnitude per unit length and with infinite length.

\section{Research aim and objectives}

The research aim is to implement the Fourier sine transform method for solving the Cerrutti problem of point load tangentially applied to the origin of an elastic half plane.

The objectives are:

i. to present a stress formulation of the Cerrutti problem of elastic half plane as a biharmonic differential equation in terms of stress potential functions. 
ii. to apply the Fourier transform to the biharmonic stress compatibility equation, and obtain bounded stress functions for the Cerrutti problems of the elastic half plane.

iii. to express the stresses and strains in terms of the stress functions and obtain the stress fields and strain fields in the Fourier sine transform space.

iv. to use the boundary conditions and obtain the unknown constants of the stress function.

v. to apply the inverse transformation to the stresses and strains in the transform space and obtain the stresses and strains in the physical domain space variables.

\section{Research problem: The Cerrutti's problem in two dimensions ( $x z$ plane)}

The Cerrutti's problem in two dimensional (2D) space is the problem of determining the stress fields and displacement fields at any arbitrary point $A(x, z)$ or $A(r, \theta)$ due to a single tangential point load of magnitude $Q_{0}$ acting at the origin $(0,0)$ on the surface of a linear elastic half space material (soil) of semi-infinite extent in the Cartesian coordinate $(x, z)$ space, where $-\infty \leq x \leq \infty$; $y=0 ; 0 \leq z \leq \infty$. The problem is illustrated graphically in Figure 1.

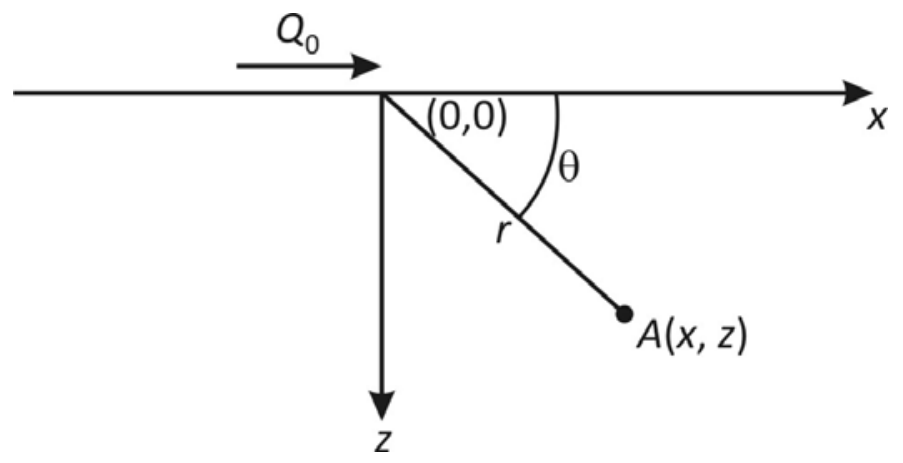

Fig. 1 - Cerrutti's problem of the elastic half plane

The point load $Q_{0}$ is assumed to act in the direction of the positive x-axis as shown in Figure 1. $r$ and $\theta$ are radial and tangential coordinates of the 2D polar coordinate system, and $r^{2}=x^{2}+z^{2}$, $\tan \theta=\frac{z}{x}$.

\section{Theoretical framework}

Formulation of the Cerrutti problem using Boussinesq-Papkovich displacement potential functions

We define the Boussinesq-Papkovich displacement potential functions $\phi(x, z)$ and $\psi(x, z)$ as scalar fields of the coordinates of the two dimensional (2D) Cartesian space, from which the displacements are derivable as follows:

$$
\begin{aligned}
& u(x, z)=\frac{-1}{4(1-\mu)} \frac{\partial \phi}{\partial x}(x, z)=u_{x}(x, z) \\
& w(x, z)=u_{z}(x, z)=\psi(x, z)-\frac{1}{4(1-\mu)} \frac{\partial \phi}{\partial z}
\end{aligned}
$$

where $u=u_{x}$ is the displacement field component in the $x$ direction, $w=u_{z}$ is the displacement field component in the $z$ direction; and $\mu$ is the Poisson's ratio of the elastic half plane material (soil). 
Elastic half plane problems satisfy simultaneously the fundamental equations of elasticity namely: strain - displacement relations (kinematic relations), Hooke's generalised stress - strain equations, and the differential equations of equilibrium together with the boundary conditions $[3-5,15]$.

\section{Strain fields}

For infinitesimal (small) displacement assumptions in the theory of elasticity, the normal and shear strain fields $\varepsilon_{x x}, \varepsilon_{z z}$ and $\gamma_{x z}$ are found from the strain displacement relations of the theory of small displacement elasticity as follows:

$$
\begin{aligned}
& \varepsilon_{x x}=\frac{\partial u_{x}}{\partial x}=\frac{\partial}{\partial x}\left(\frac{-1}{4(1-\mu)} \frac{\partial \phi}{\partial x}\right)=\frac{-1}{4(1-\mu)} \frac{\partial^{2} \phi}{\partial x^{2}} \\
& \varepsilon_{z z}=\frac{\partial w}{\partial z}=\frac{\partial u_{z}}{\partial z}=\frac{\partial}{\partial z}\left(\psi-\frac{1}{4(1-\mu)} \frac{\partial \phi}{\partial z}\right) \\
& \varepsilon_{z z}=\frac{\partial \psi}{\partial z}-\frac{1}{4(1-\mu)} \frac{\partial^{2} \phi}{\partial z^{2}} \\
& \gamma_{x z}=\frac{\partial u_{x}}{\partial z}+\frac{\partial u_{z}}{\partial x}=\frac{\partial u_{x}}{\partial z}+\frac{\partial w}{\partial x} \\
& \gamma_{x z}=\frac{\partial}{\partial z}\left(\frac{-1}{4(1-\mu)} \frac{\partial \phi}{\partial x}\right)+\frac{\partial}{\partial x}\left(\psi-\frac{1}{4(1-\mu)} \frac{\partial \phi}{\partial z}\right) \\
& \gamma_{x z}=\frac{\partial \psi}{\partial x}-\frac{1}{2(1-\mu)} \frac{\partial^{2} \phi}{\partial x \partial z} \\
& \varepsilon_{x z}=\frac{1}{2} \gamma_{x z}=\frac{-1}{4(1-\mu)}\left(\frac{\partial^{2} \phi}{\partial x \partial z}-2(1-\mu) \frac{\partial \psi}{\partial x}\right)
\end{aligned}
$$

\section{Stress fields}

The generalized Hooke's stress - strain laws expressed in terms of Lamé's constants are used to find the stress fields for plane strain conditions. The stress - strain laws are:

$$
\begin{aligned}
& \sigma_{x x}=2 G \varepsilon_{x x}+\lambda \varepsilon_{v} \\
& \sigma_{z z}=2 G \varepsilon_{z z}+\lambda \varepsilon_{v} \\
& \tau_{x z}=G \gamma_{x y}=2 G \varepsilon_{x y}
\end{aligned}
$$

where $\varepsilon_{v}$ is the volumetric strain, $\sigma_{x x}$ and $\sigma_{z z}$ are normal stresses, $\tau_{x z}$ is the shear stress, $G$ is the shear modulus.

$\lambda$ is the Lamé's constant given by:

$$
\lambda=\frac{2 \mu G}{1-2 \mu}
$$

The volumetric strain $\varepsilon_{v}$ can be obtained in terms of the Boussinesq-Papkovich functions as:

$$
\varepsilon_{v}=\frac{1-2 \mu}{2(1-\mu)} \frac{\partial \psi}{\partial z}
$$

Then, the stress fields are obtained in terms of the Boussinesq-Papkovich harmonic functions as:

$$
\begin{aligned}
& \sigma_{x x}=\frac{-G}{2(1-\mu)} \frac{\partial^{2} \phi}{\partial x^{2}}+\frac{2 \mu G}{2(1-\mu)} \frac{\partial \psi}{\partial z} \\
& \sigma_{x x}=\frac{-G}{2(1-\mu)}\left(\frac{\partial^{2} \phi}{\partial x^{2}}-2 \mu \frac{\partial \psi}{\partial z}\right)
\end{aligned}
$$




$$
\begin{aligned}
\sigma_{z z} & =2 G\left(\frac{\partial \psi}{\partial z}-\frac{1}{4(1-\mu)} \frac{\partial^{2} \phi}{\partial z^{2}}\right)+\frac{\mu G}{1-\mu} \frac{\partial \psi}{\partial z} \\
\sigma_{z z} & =\frac{-G}{2(1-\mu)}\left(\frac{\partial^{2} \phi}{\partial z^{2}}-2(2-\mu) \frac{\partial \psi}{\partial z}\right) \\
\tau_{x z} & =\frac{-G}{2(1-\mu)}\left(\frac{\partial^{2} \phi}{\partial x \partial z}-2(1-\mu) \frac{\partial \psi}{\partial x}\right)
\end{aligned}
$$

We observe that the Boussinesq-Papkovich displacement potential functions $\phi$ and $\psi$ can also be termed stress potential functions since the stress fields are also derivable from the functions by Equations (16), (18) and (19).

\section{Results}

\section{Stress formulation of the boundary value problem}

The governing partial differential equation for the elasticity problem involving the half plane is given by the stress function formulation which is a biharmonic equation in $\phi(x, z)$ as:

$$
\nabla^{4} \phi(x, z)=\nabla^{2} \nabla^{2} \phi(x, z)=0
$$

where $\phi(x, z)$ is the stress function which must be bounded within the half space region; and

$$
\nabla^{4}=\nabla^{2} \nabla^{2}
$$

$\nabla^{2}$ is the Laplacian operator

$\nabla^{4}$ is the biharmonic operator.

$$
\begin{aligned}
& \nabla^{2}=\frac{\partial^{2}}{\partial x^{2}}+\frac{\partial^{2}}{\partial z^{2}} \\
& \nabla^{4}=\left(\frac{\partial^{2}}{\partial x^{2}}+\frac{\partial^{2}}{\partial z^{2}}\right)^{2}=\frac{\partial^{4}}{\partial x^{4}}+\frac{2 \partial^{4}}{\partial x^{2} \partial z^{2}}+\frac{\partial^{4}}{\partial z^{4}}
\end{aligned}
$$

\section{Solution for a suitable stress function}

We seek a solution for a suitable stress function by solving the biharmonic equation for $\phi(x, z)$ subject to the boundary conditions and boundedness requirements. We apply the Fourier sine transformation with respect to the $x$-coordinate to the biharmonic equation to obtain:

$$
\begin{aligned}
& \frac{1}{\sqrt{2 \pi}} \int_{-\infty}^{\infty}\left(\frac{\partial^{4} \phi}{\partial x^{4}}+2 \frac{\partial^{4} \phi}{\partial x^{2} \partial z^{2}}+\frac{\partial^{4} \phi}{\partial z^{4}}\right) \operatorname{Re}^{-i k x} d x=0 \\
& \frac{1}{\sqrt{2 \pi}} \int_{-\infty}^{\infty}\left(\frac{\partial^{4} \phi}{\partial x^{4}}+2 \frac{\partial^{4} \phi}{\partial x^{2} \partial z^{2}}+\frac{\partial^{4} \phi}{\partial z^{4}}\right) \sin k x d x=0 \\
& k^{4} \bar{\phi}-2 k^{2} \frac{d^{2} \bar{\phi}}{d z^{2}}+\frac{d^{4} \bar{\phi}}{d z^{4}}=0
\end{aligned}
$$

where $k$ is the Fourier transform parameter (also, the Fourier sine transform parameter).

Where $\bar{\phi}=\bar{\phi}(k, z)=\frac{1}{\sqrt{2 \pi}} \int_{-\infty}^{\infty} \phi(x, z) e^{-i k x} d x$

or $\bar{\phi}(k, z)=\int_{-\infty}^{\infty} \phi(x, z) \sin k x d x$ 
$\bar{\phi}(k, z)$ is the Fourier transform of the stress function or the Fourier sine transformation of the stress function.

Equation (26) is a fourth order ordinary homogeneous differential equation in $\bar{\phi}(k, z)$.

Solution using the method of trial functions, $D$ operator techniques or auxiliary polynomial methods yield the solution as:

$$
\bar{\phi}(k, z)=\left(c_{1}+c_{2} k z\right) \exp (-k z)+\left(c_{3}+c_{4} k z\right) \exp (k z)
$$

where $c_{1}, c_{2}, c_{3}$ and $c_{4}$ are the four constants of integration.

For bounded stress functions, $\bar{\phi}(k, z)$ should be bounded and definite as $z \rightarrow \infty$. This is satisfied only when

$\begin{aligned} c_{3} & =0 \\ \text { and, } \quad c_{4} & =0\end{aligned}$

Thus,

$$
\begin{aligned}
& \bar{\phi}(k, z)=\left(c_{1}+c_{2} k z\right) \exp (-k z) \\
& \bar{\phi}(k, z)=\left(c_{1}+c_{2} k z\right) e^{-k z}
\end{aligned}
$$

The Boussinesq-Papkovich stress (or displacement) potential functions in the Fourier transform space can then be defined as:

$$
\bar{\psi}(k, z)=c_{2} k e^{-k z}
$$

and $\bar{\phi}(k, z)$ given by Equation (33).

\section{Application of the Fourier sine transformation}

The Fourier sine transformation is applied to the Boussinesq-Papkovich potential functions already obtained in the Fourier transform space, to obtain:

$$
\begin{aligned}
& \bar{\phi}(k, z)=\int_{0}^{\infty}\left(c_{1}+c_{2} k z\right) e^{-k z} \sin k x d k \\
& \bar{\psi}(k, z)=\int_{0}^{\infty} c_{2} k e^{-k z} \sin k x d k \\
& \text { where } c_{1}=c_{1}(k) \\
& c_{2}=c_{2}(k) \\
& 0 \leq k \leq \infty
\end{aligned}
$$

and $k$ is the Fourier integral sine transform parameter.

Similarly, the Fourier sine integral transformation is applied to the boundary conditions to yield:

$$
\begin{aligned}
& \sigma_{z z}(x, z=0)=0 \\
& \int_{0}^{\infty} \sigma_{z z}(x, z=0) \sin k x d k=0 \\
& \tau_{x z}(x, z=0)=Q_{0} \delta(x) \\
& \int_{0}^{\infty} \tau_{x z}(x, z=0) \sin k x d k=\bar{\tau}_{x z}(k, z=0)
\end{aligned}
$$




$$
\begin{aligned}
& =\frac{1}{2 \pi} \int_{0}^{\infty} Q_{0} \delta(x) \cos k x d k \\
& =-\frac{Q_{0}}{\pi} \int_{0}^{\infty} \cos k x d k
\end{aligned}
$$

Application of the Fourier sine integral transformation to the stress fields yield:

$$
\begin{aligned}
& \bar{\sigma}_{x x}(k, z)=\frac{-G}{2(1-\mu)} \int_{0}^{\infty}\left(\frac{\partial^{2} \bar{\phi}}{\partial x^{2}}-2 \mu \frac{\partial \bar{\psi}}{\partial z}\right) \sin k x d k \\
& \bar{\sigma}_{z z}(k, z)=\frac{-G}{2(1-\mu)} \int_{0}^{\infty}\left(\frac{\partial^{2} \phi}{\partial z^{2}}-2(2-\mu) \frac{\partial \psi}{\partial z}\right) \sin k x d k \\
& \bar{\tau}_{z z}(k, z)=\frac{-G}{2(1-\mu)} \int_{0}^{\infty}\left(\frac{\partial^{2} \phi}{\partial x \partial z}-2(1-\mu) \frac{\partial \psi}{\partial x}\right) \sin k x d k
\end{aligned}
$$

where $\bar{\sigma}_{x x}(k, z), \bar{\sigma}_{z z}(k, z)$ and $\bar{\tau}_{x z}(k, z)$ are the stress fields in the Fourier sine transform space.

Substitution of expressions for $\phi$ and $\psi$ and evaluation of partial derivatives yield, after simplification:

$$
\begin{aligned}
& \left.\bar{\sigma}_{x x}(k, z)=\frac{G}{2(1-\mu)} \int_{0}^{\infty}\left(-c_{1}+2 \mu-k z\right) c_{2}\right) k^{2} e^{-k z} \sin k x d k \\
& \bar{\sigma}_{z z}(k, z)=\frac{G}{2(1-\mu)} \int_{0}^{\infty}\left(c_{1}+(2(1-\mu)+k z) c_{2}\right) k^{2} e^{-k z} \sin k x d k \\
& \bar{\sigma}_{z z}(k, z=0)=\frac{G}{2(1-\mu)} \int_{0}^{\infty}\left(c_{1}+2(1-\mu) c_{2}\right) k^{2} \sin k x d k \\
& \bar{\tau}_{x z}(k, z)=\frac{G}{2(1-\mu)} \int_{0}^{\infty}\left(c_{1}+c_{2}(1-2 \mu+k z)\right) k^{2} e^{-k z} \cos k x d k \\
& \bar{\tau}_{x z}(k, z=0)=\frac{G}{2(1-\mu)} \int_{0}^{\infty}\left(c_{1}+c_{2}(1-2 \mu)\right) k^{2} \cos k x d k \\
& =-\frac{Q_{0}}{\pi} \int_{0}^{\infty} \cos k x d k \\
& =\frac{G}{2(1-\mu)}\left(c_{1}+c_{2}(1-2 \mu)\right) \int_{0}^{\infty} k^{2} \cos k x d k
\end{aligned}
$$

\section{Enforcement of boundary conditions}

Application of the boundary condition on $\bar{\sigma}_{x x}$ and $\bar{\tau}_{x z}$ at $z=0$ yield the system of two equations in terms of the constants $c_{1}(k)$ and $c_{2}(k)$ as follows:

$$
\begin{aligned}
& c_{1}+2(1-\mu) c_{2}=0 \\
& \frac{G}{2(1-\mu)}\left(c_{1}+(1-2 \mu) c_{2}\right) k^{2}=\frac{-Q_{0}}{\pi}
\end{aligned}
$$

Solving using the method of elimination,

$$
c_{1}=-2(1-\mu) c_{2}
$$




$$
\begin{aligned}
& \frac{G k^{2}}{2(1-\mu)}\left((1-2 \mu) c_{2}-2(1-\mu) c_{2}\right)=\frac{-Q_{0}}{\pi} \\
& \frac{G k^{2}}{2(1-\mu)}\left(-c_{2}\right)=\frac{-Q_{0}}{\pi} \\
& c_{2}=\frac{2 Q_{0}(1-\mu)}{\pi G k^{2}}=c_{2}(k)
\end{aligned}
$$

Then, $c_{1}=\frac{-4 Q_{0}(1-\mu)^{2}}{\pi G k}=c_{1}(k)$

The constants of integration are now completely determined. The Boussinesq-Papkovich stress functions are thus completely determined as:

$$
\begin{aligned}
& \bar{\phi}(k, z)=\int_{0}^{\infty}\left(\frac{-4 Q_{0}(1-\mu)^{2}}{\pi G k^{2}}+\frac{2 Q_{0}(1-\mu) k z}{\pi G k^{2}}\right) e^{-k z} \sin k x d k \\
& \bar{\phi}(k, z)=\frac{-2 Q_{0}(1-\mu)}{\pi G} \int_{0}^{\infty}\left(\frac{2(1-\mu)-k z}{k^{2}}\right) e^{-k z} \sin k x d k
\end{aligned}
$$

The stresses are found as:

$$
\begin{aligned}
& \sigma_{x x}=\frac{-Q_{0}}{\pi} \int_{0}^{\infty}(2-k z) e^{-k z} \sin k x d k \\
& \sigma_{x x}=\frac{-2 Q_{0} x^{3}}{\pi r^{4}}=-\frac{2 Q_{0}}{\pi} \frac{x^{3}}{\left(x^{2}+z^{2}\right)^{2}}=-\frac{2 Q_{0}}{\pi x}\left(1+\frac{z^{2}}{x^{2}}\right)^{-2} \\
& \sigma_{z z}=\frac{-Q_{0}}{\pi} \int_{0}^{\infty} k e^{-k z} \sin k x d k \\
& \sigma_{z z}=\frac{-2 Q_{0}}{\pi r^{4}} x z^{2}=-\frac{2 Q_{0}}{\pi} \frac{x z^{2}}{\left(x^{2}+z^{2}\right)^{2}}=-\frac{2 Q_{0}}{\pi} \frac{x}{z^{2}}\left(1+\left(\frac{x}{z}\right)^{2}\right)^{-2} \\
& \tau_{x z}=\frac{-Q_{0}}{\pi} \int_{0}^{\infty}(1-k z) e^{-k z} \cos k x d k \\
& \tau_{x z}=\frac{-2 Q_{0} x^{2} z}{\pi r^{4}}=-\frac{2 Q_{0}}{\pi} \frac{x^{2} z}{\left(x^{2}+z^{2}\right)^{2}}=\frac{-2 Q_{0} z}{\pi x^{2}}\left(1+\frac{z^{2}}{x^{2}}\right)^{-2}
\end{aligned}
$$

The stresses are the same as the stresses found for the same problem by Bryant [16].

\section{Strains}

The strains are obtained as follows:

$$
\begin{aligned}
& \varepsilon_{x x}=\frac{-1}{4(1-\mu)} \frac{\partial^{2}}{\partial x^{2}}\left(\frac{-2 Q_{0}(1-\mu)}{\pi G} \int_{0}^{\infty}\left(\frac{2(1-\mu)-k z}{k^{2}}\right)\right) e^{-k z} \sin k x d k \\
& \varepsilon_{x x}=\frac{-Q_{0}}{2 \pi G} \int_{0}^{\infty}(2(1-\mu)-k z) e^{-k z} \sin k x d k \\
& \varepsilon_{x x}=\frac{-Q_{0} x}{\pi G r^{2}}\left(1-\mu-\frac{z^{2}}{r^{2}}\right)=\frac{-Q_{0}}{\pi G} \frac{x}{\left(x^{2}+z^{2}\right)}\left(1-\mu-\frac{z^{2}}{x^{2}+z^{2}}\right)
\end{aligned}
$$




$$
\begin{aligned}
& \varepsilon_{z z}=\frac{-2 Q_{0}(1-\mu)}{\pi G} \int_{0}^{\infty} e^{-k z} \sin k x d k-\frac{1}{4(1-\mu)}\left(-\frac{2 Q_{0}(1-\mu)}{\pi G}\right) \int_{0}^{\infty}(2(1-\mu)+(2-k z)) e^{-k z} \sin k x d k \\
& \varepsilon_{z z}=\frac{Q_{0}}{2 \pi G} \int_{0}^{\infty}(2 \mu-k z) e^{-k z} \sin k x d k \\
& \varepsilon_{z z}=\frac{Q_{0} x}{\pi G r^{2}}\left(\mu-\frac{z^{2}}{r^{2}}\right)=\frac{Q_{0}}{\pi G} \frac{x}{\left(x^{2}+z^{2}\right)}\left(\mu-\frac{z^{2}}{x^{2}+z^{2}}\right) \\
& \varepsilon_{x z}=\frac{-1}{4(1-\mu)} \cdot \frac{2 Q_{0}(1-\mu)}{\pi G} \int_{0}^{\infty}(2(1-\mu)+1-k z) e^{-k z} \cos k x d k+\frac{1}{2} \frac{2 Q_{0}(1-\mu)}{\pi G} \int_{0}^{\infty} e^{-k z} \cos k x d k \\
& \varepsilon_{x z}=\frac{-Q_{0}}{2 \pi G} \int_{0}^{\infty}(1-k z) e^{-k z} \cos k x d k \\
& \varepsilon_{x z}=\frac{-Q_{0} x^{2} z}{\pi G r^{4}}=\frac{-Q_{0}}{\pi G} \frac{x^{2} z}{\left(x^{2}+z^{2}\right)^{2}}
\end{aligned}
$$

\section{Displacement field}

The integration problem involved in finding or solving for the $x$ component of the displacement field $\left(u_{x}\right)$ from the Boussinesq-Papkovich definition of the displacement potential function namely Equation (1) would result in a divergent integral.

However, the $x$ component of the displacement field $u_{x}$ is obtained from the simultaneous application of the expressions for $\varepsilon_{x x}$ and the strain - displacement relation. Thus, we obtain the problem:

$$
\begin{aligned}
& \varepsilon_{x x}=\frac{\partial u_{x}}{\partial x}=\frac{-Q_{0}}{2 \pi G} \int_{0}^{\infty}(2(1-\mu)-k z) e^{-k z} \sin k x d k \\
& \left.\partial u_{x}=\frac{-Q_{0}}{2 \pi G} \int_{0}^{\infty} 2(1-\mu)-k z\right) e^{-k z} \sin k x d k d x
\end{aligned}
$$

Integration of Equation (80) with respect to the variable, $x$ over the interval $(0, x)$ yields:

$$
\begin{aligned}
& u_{x}(x, z)-u_{x}(0, z)=\frac{-Q_{0}}{2 \pi G} \int_{0}^{\infty} \int_{0}^{x}(2(1-\mu)-k z) e^{-k z} \sin k x d x d k \\
& u_{x}(x, z)-u_{x}(0, z)=\frac{-Q_{0}}{2 \pi G} \int_{0}^{\infty}(2(1-\mu)-k z) e^{-k z}\left(\frac{1-\cos k x}{k}\right) d k \\
& u_{x}(x, z)=\frac{Q_{0}}{2 \pi G}\left(2(1-\mu) \ln \frac{z}{r}+\left(1-\frac{z^{2}}{r^{2}}\right)\right)=\frac{Q_{0}}{2 \pi G}\left(2(1-\mu) \ln \frac{z}{\sqrt{\left(x^{2}+z^{2}\right)}}+\left(1-\frac{z^{2}}{x^{2}+z^{2}}\right)\right) \\
& u_{x}(x, z)=\frac{Q_{0}(1+\mu)}{\pi E}\left(2(1-\mu) \ln \frac{z}{r}+\left(1-\frac{z^{2}}{r^{2}}\right)\right)=\frac{Q_{0}(1+\mu)}{\pi E}\left(2(1-\mu) \ln \frac{z}{\sqrt{\left(x^{2}+z^{2}\right)}}+\left(1-\frac{z^{2}}{x^{2}+z^{2}}\right)\right) \\
& =\frac{Q_{0}(1+\mu)}{\pi E}\left(2(1-\mu) \ln \frac{z}{\sqrt{\left(x^{2}+z^{2}\right)}}+\frac{x^{2}}{x^{2}+z^{2}}\right)
\end{aligned}
$$


Similarly, from

$$
\begin{aligned}
& \varepsilon_{z z}=\frac{\partial u_{z}}{\partial z}=\frac{Q_{0}}{2 \pi G} \int_{0}^{\infty}(2 \mu-k z) e^{-k z} \sin k x d k \\
& d u_{z}=\frac{Q_{0}}{2 \pi G} \int_{0}^{\infty}(2 \mu-k z) e^{-k z} \sin k x d k d z
\end{aligned}
$$

Integration with respect to $z$ yields:

$$
\begin{aligned}
& u_{z}(x z)-u_{z}(0, z)=\frac{Q_{0}}{2 \pi G} \int_{0}^{\infty} \int_{0}^{z}(2 \mu-k z) e^{-k z} \sin k x d k d z \\
& =\frac{Q_{0}}{2 \pi G} \int_{0}^{\infty}(1-2 \mu+k z) \frac{e^{-k z} \sin k x}{k} d k \\
& u_{z}(x, z)=\frac{Q_{0}}{2 \pi G}\left((1-2 \mu) \tan ^{-1} \frac{x}{z}+\frac{x z}{r^{2}}\right)=\frac{Q_{0}}{2 \pi G}\left((1-2 \mu) \tan ^{-1}\left(\frac{x}{z}\right)+\frac{x z}{\left(x^{2}+z^{2}\right)}\right) \\
& u_{z}(x, z)=\frac{Q_{0} 2(1+\mu)}{2 \pi E}\left((1-2 \mu) \tan ^{-1} \frac{x}{z}+\frac{x z}{r^{2}}\right)=\frac{Q_{0} 2(1+\mu)}{2 \pi E}\left((1-2 \mu) \tan ^{-1} \frac{x}{z}+\frac{x z}{x^{2}+z^{2}}\right) \\
& u_{z}(x, z)=\frac{Q_{0}(1+\mu)}{\pi E}\left((1-2 \mu) \tan ^{-1} \frac{x}{z}+\frac{x z}{r^{2}}\right)=\frac{Q_{0}(1+\mu)}{\pi E}\left((1-2 \mu) \tan ^{-1} \frac{x}{z}+\frac{x z}{x^{2}+z^{2}}\right)
\end{aligned}
$$

where, $r^{2}=x^{2}+z^{2}$

\section{Discussion}

In this study, the Fourier sine transformation method has been successfully implemented to solve the problem of point load $Q_{0}$ acting at the origin $O$ and applied tangentially in the positive $x$ direction to an elastic half plane; commonly called the Cerrutti problem. The soil occupying the half plane region was assumed homogeneous, isotropic and linear elastic. Stress based formulation of the half plane problem was used. The Boussinesq-Papkovich potential functions, defined as Equations (1) and (2) were used to express the strains as Equations (3), (5) and (8). The stresses were similarly expressed as Equations (16), (18) and (19).

The method of Fourier transformation or Fourier sine transformation was applied to the biharmonic stress compatibility equation to obtain the solution for the stress functions as Equation (29). Boundedness criteria was applied to obtain bounded stress function as Equation (33) and (34). The Fourier sine transform was similarly used to obtain the stress fields in the Fourier sine transform space as Equations $(45-47)$. The stresses were obtained in the Fourier sine transform space in terms of the Boussinesq-Papkovich stress functions as Equations (48), (50) and (51). Boundary conditions of stresses at $z=0$ were used to find the unknown constants of the stress functions as Equations (60) and (61). This led to the complete determination of the stresses as Equations (65 - 69). The strains were obtained as Equations (71 - 78). Displacements were obtained by integration of the strain displacement equations, as Equations (84) and (89). It 
was observed that the expressions obtained for the stresses and displacements are identical to the solutions found in the technical literature.

\section{Conclusions}

The conclusions of the study are as follows:

i. The Fourier sine transform method is an effective technique for solving the Cerrutti problem for a linear elastic, isotropic homogeneous half plane.

ii. The Fourier sine transformation operation simplifies the governing stress compatibility equation, which is a fourth order biharmonic partial differential equation (PDE) in terms of the stress function to a linear fourth order ordinary differential equation with constant coefficients; which is more easily amenable to analytical solutions than the previously untransformed biharmonic equation.

iii. The Boussinesq-Papkovich stress potential function formulation of the Cerrutti problem simplified the solution process to finding suitable functions that solve the biharmonic stress compatibility equation, and simultaneously satisfied the traction and boundary conditions as well as the boundedness requirements for stresses and displacements.

\section{References}

[1] Apostol B.F. (2017). Elastic Displacement in a Half-Space under the action of a tensor force, General Solution for the Half Space with Point Forces, Journal of Elasticity Volume 126 pp. 231 - 244. DOI:10.1007/s10659-016-9592-3.

[2] Apostol B.F. (2016). Elastic Equilibrium of the Half Space revisited, Mindlin and Boussinesq Problems, Journal of Elasticity. DOI: 10.1007/s10659-016-9574-5.

[3] Nwoji C.U., Onah H.N., Mama B.O. and Ike C.C. (2017). Solution of the Boussinesq problem of half space using Green and Zerna displacement potential function method. The Electronic Journal of Geotechnical Engineering (EJGE) 22. 11 pp. 4305 - 4314, Available at ejge.com.

[4] Ike C.C., Mama B.O., Onah H.N. and Nwoji C.U. (2017). Tefftz Harmonic function method for solving Boussinesq problem. Electronic Journal of Geotechnical Engineering, (22.12) pp 4589 - 4601. Available at ejge.com.

[5] Nwoji C.U., Onah H.N., Mama B.O. and Ike C.C. (2017). Solution of elastic half space problem using Boussinesq displacement potential functions. Asian Journal of Applied Sciences (AJAS) Vol 5 No. 5. pp 1100 - 1106, October, 2017.

[6] Chan K.T. (2013). Analytic Methods in Geomechanics CRC Press Taylor and Francis Group, New York.

[7] Sadd M.H. (2014). Elasticity Theory Applications and Numerics, Third Edition, Elsevier Academic Press Amsterdam.

[8] Lurie S.A. \& Vasilev V.V. (1995). The Biharmonic Problem in the Theory of Elasticity. Gordon and Breach Publishers, United States, United Kingdom.

[9] Sitharam T.G. \& L. Govinda Reju L. (2017). Applied Elasticity for Engineers Module: Elastic Solutions and Applications in Geomechanics 14.139.172.204/nptel/1/CSE/web/ 10.5108070/module8/lecture 17/pdf.

[10] Hazel A. (2015). MATH 350211 Elasticity. www.maths.manchester.ac.uk/a hazel/MATHS. Nov 30, 2015.

[11] Palaniappan D. (2011). A general solution of equations of equilibrium in linear elasticity. Applied Mathematical Modelling, Elsevier 35 pp. 5494 - 5499.

[12] Padio Guidugli P. \& and Favata A. (2014). The Cerrutti Problem: Elasticity for Geotechnicians. Solid Mechanics and its Applications SMIA 2014. Springer International Publishing, Switzerland. Pp 149 - 157.

[13] Teodorescu P.P. (2013). Treatise on Classical Elasticity Theory and Related Problems. Mathematical and Analytical Techniques with Applications to Engineering. Springer Dordrecht. DOI:10.1007/978-94-007-2616-1.

[14] Love A.E. (1929). The stress produced in a semi-infinite solid by pressure on part of the boundary. Philosophical transactions of the Royal Society A Vol 228 No 659 - 669 pp 377 - 420.

[15] Ike C.C. (2006). Principles of Soil Mechanics, De-Adroit Innovation, Enugu.

[16] Bryant (2006). ME383s Contact www.me.utexas.edu/ bryant/courses/me383s/Download Files/Lecture Notes/Contact. 